\title{
Downregulated IncRNA SNHG I8 Suppresses the Progression of Hepatitis B Virus-Associated Hepatocellular Carcinoma and Meditates the Antitumor Effect of Oleanolic Acid
}

\author{
Wenqi Song ${ }^{1}, *$, Xuemei Zhang ${ }^{2, *}$, Lin Feng', Yongwei Lai', Tongtong Li', Pengxia Zhang' \\ ' Key Laboratory of Microecology-immune Regulatory Network and Related Diseases, School of Basic Medicine, Jiamusi University, Jiamusi, \\ Heilongjiang, People's Republic of China; ${ }^{2}$ Department of Gastroenterology, The First Affiliated Hospital of Jiamusi University, Jiamusi, Heilongjiang, \\ People's Republic of China \\ *These authors contributed equally to this work
}

Correspondence: Pengxia Zhang, Key Laboratory of Microecology-immune Regulatory Network and Related Diseases, School of Basic Medicine, Jiamusi University, I 48 Xuefu Street, Jiamusi, Heilongjiang, I54000, People’s Republic of China, Email zhangpengxiajms@I63.com

\begin{abstract}
Purpose: Oleanolic acid (OA) has been widely reported to possess antitumor effects, but the specific molecular mechanism underlying its inhibition of hepatocellular carcinoma (HCC) progression remains unclear. This study aims to uncover the mechanism of OA antitumor effect on HBV-associated HCC and identify a potential biomarker for tumor progression.

Patients and Methods: The effect of OA on major cellular processes of HBV-associated HCC cells was evaluated by CCK8 and Transwell assay. The potential molecular mechanism was assessed by cell transfection. This study also enrolled 111 HCC patients infected with HBV to evaluate the prognostic potential of lncRNA SNHG18 (SNHG18) in HBV-associated HCC.

Results: The inhibitory effect of OA was observed in the critical cellular processes of HBV-associated HCC cells, which depend on OA concentration. Downregulated SNHG18 in HBV-associated HCC was demonstrated to be involved in disease development and predict patients' prognosis. The downregulation of SNHG18 dramatically promoted cellular processes of HBV-associated HCC could reverse the inhibitory effect of OA.
\end{abstract}

Conclusion: SNHG18 served as a tumor suppressor and prognostic biomarker of HBV-associated HCC. Enhancing SNHG18 might be the mechanism underlying the antitumor effect of OA in HBV-associated HCC.

Keywords: HBV, HCC, oleanolic acid, lncRNA SNHG18, prognosis, progression

\section{Introduction}

Hepatocellular carcinoma (HCC) is a frequent type of liver cancer and is also one of the most common causes of cancerrelated mortality. Chronic hepatitis B virus (HBV) infection is closely correlated with $\mathrm{HCC}$ onset and progression. ${ }^{1}$ Over $80 \%$ of HCC patients are HBV carriers in China. ${ }^{2}$ The lack of specific symptoms results in the hysteresis of HBVassociated HCC diagnosis and the unsatisfying 5-year overall survival of patients. Currently, the screening of alphafetoprotein (AFP) is the major detection of HCC, however, the sensitivity of AFP was not enough to distinguish HCC, especially for the patients with negative AFP or low AFP levels. ${ }^{3,4}$ Therefore, it is of an urgent need to explore accessorial molecules that could indicate the onset and development of HCC.

Oleanolic acid (OA), a pentacyclic triterpenoid, has been demonstrated to be widely distributed in various herbs and possessed several pharmacological activities, including anti-inflammation and antitumor. ${ }^{5}$ For example, the inhibitory effect of OA on tumor progression of osteosarcoma was observed and was speculated to be a result of regulating mTOR signaling. ${ }^{6}$ In HCC, OA was previously reported to suppress epithelial-mesenchymal transition and induce cell apoptosis and autophagy. ${ }^{7,8}$ The mechanism investigation would benefit the clinical application of OA in tumor treatment. 


\section{Graphical Abstract}

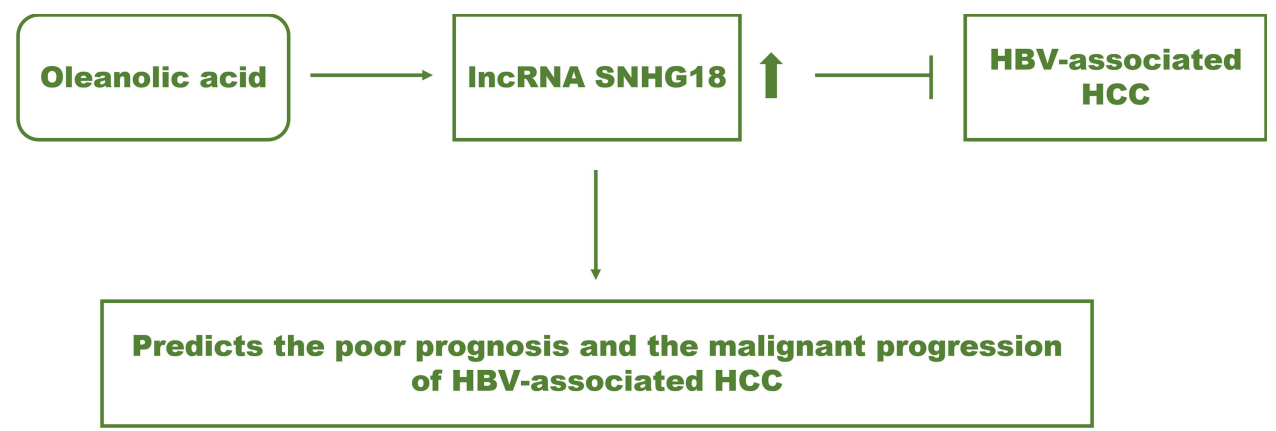

It has been reviewed that the development of HBV-associated HCC involved a variety of molecules, including differently expressed non-coding RNAs. ${ }^{9}$ Previously, regulating corresponding molecular biomarkers is always considered as the pathway of the different active ingredients in herbs during their antitumor effects. OA was reported to cause the elevated expression of miR-122 in non-small cell lung cancer, which enhanced the suppression of cell proliferation by OA. ${ }^{10}$ Increasing interests have been paid to long non-coding RNAs (lncRNAs) in recent tumor research, which illustrated its significant role in disease diagnosis, prognosis, and development of human cancers as well as the pharmacological effect of various drugs or herbs. For instance, the alleviated effect of OA on arteriosclerosis was revealed to result from the elevation of lincRNA-p21. ${ }^{11}$ IncRNA SNHG18 (SNHG18) was reported to be a potential diagnosis indicator of HCC with a significant downregulation compared with normal samples. ${ }^{12}$ The involvement of SNHG18 has been reported in various malignant tumors, such as glioma and myeloma. ${ }^{13,14}$ However, the specific function of SNHG18 in HBV-associated HCC and whether it mediated the antitumor effect of OA remains unclear.

Here, the function of SNHG18 in HBV-associated HCC was investigated and evaluated its role during the inhibition of tumor progression by OA.

\section{Materials and Methods}

\section{Cell Culture}

Four HCC cell lines with an HBV transformant, HepG2/2.2.15, SNU182, MHCC97H, PLC8024, and a normal human liver cell L02 were obtained from ATCC. The DMEM culture medium (Invitrogen, USA) with 10\% FBS was used for the incubation of HepG2.2.15, MHCC97H, PLC8024, and L02 cells, while the SNU182 cell was cultured in the RPMI 1640 culture medium (GIBCO, USA). Cell incubation was conducted at $37^{\circ} \mathrm{C}$ with $5 \% \mathrm{CO}_{2}$.

\section{Cell Treatment and Cell Transfection}

After $24 \mathrm{~h}$ of cell culture, the cultured cells were treated with $30 \mu \mathrm{g} / \mathrm{mL}$ OA or transfected with pcDNA3.1-SNHG18 with the employment of Lipofectamine 2000 (Invitrogen, USA). The transfection efficiency was evaluated by SNHG18 expression with quantitative PCR.

\section{Cell Proliferation Assessment}

The proliferation of treated cells $\left(5 \times 10^{4}\right.$ cells/well $)$ was assessed in the 96-well plates supplied with a completed corresponding culture medium incubating at $37^{\circ} \mathrm{C}$ for $0,24,48$, and $72 \mathrm{~h}$ followed by adding CCK 8 (Dojindo, Japan) to each well. OD450 of each well was detected with a microplate reader as the indicator of cell proliferation. 


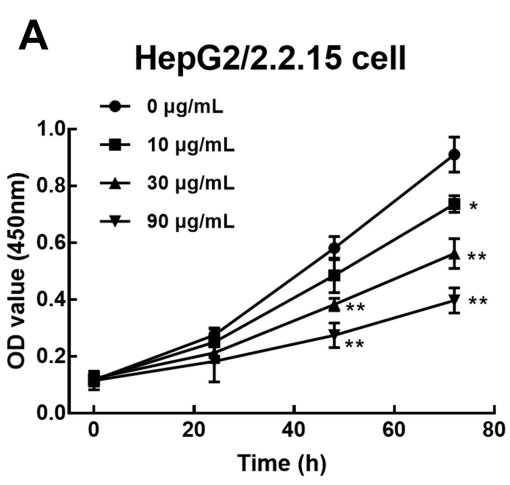

MHCC97H cell

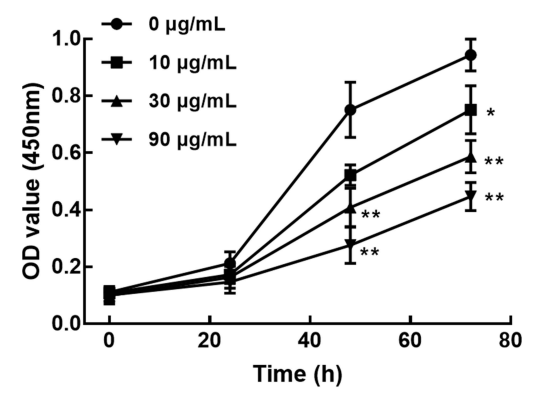

B

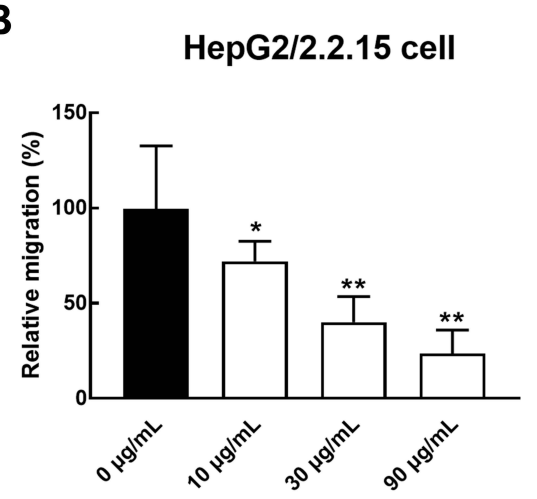

C

HepG2/2.2.15 cell

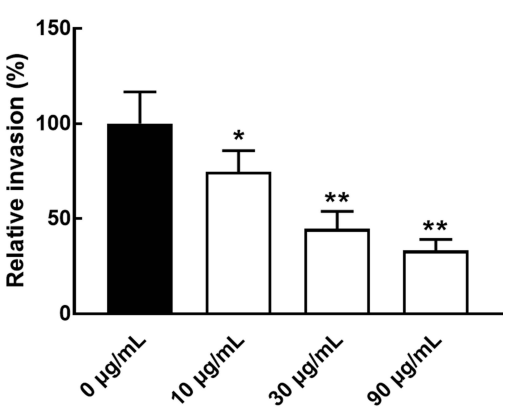

MHCC97H cell

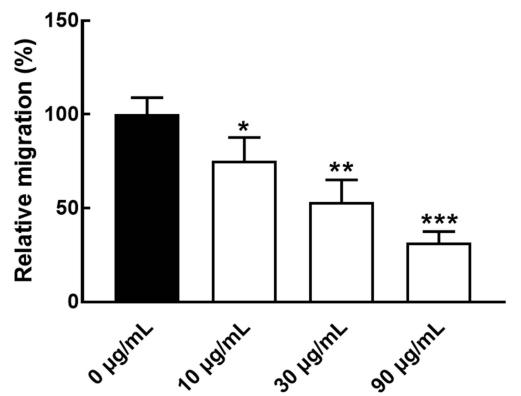

MHCC97H cell

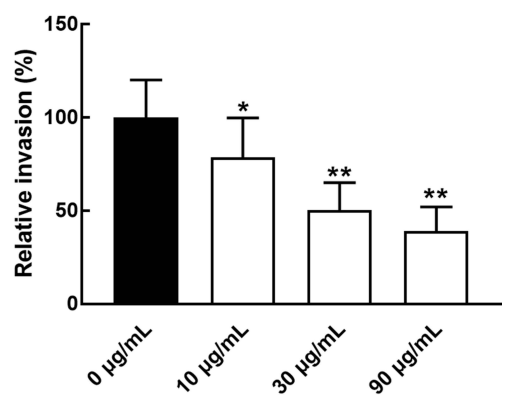

Figure I OA showed significant antitumor effects on HBV-associated HCC cells. The proliferation (A), migration (B), and invasion (C) of HepG2/2.2.15 and HCC97H cells were significantly suppressed by $\mathrm{OA}$, and the inhibition was enhanced with the increase of OA concentration. $* p<0.05$, $* * p<0.0 \mathrm{I}, * * p<0.00 \mathrm{I}$.

\section{Cell Migration and Invasion Evaluation}

Treated cells $\left(5 \times 10^{4}\right.$ cells/well) were seeded into the upper chambers of the 24-well plates (Matrigel chambers for invasion evaluation, Corning, USA). The seeded cells were supplied with an FBS-free culture medium and maintained at $37^{\circ} \mathrm{C}$ for $24 \mathrm{~h}$. Then, the cells on the bottom surface were fixed with and stained for $30 \mathrm{~min}$. The number of cells was evaluated with a microscope.

A

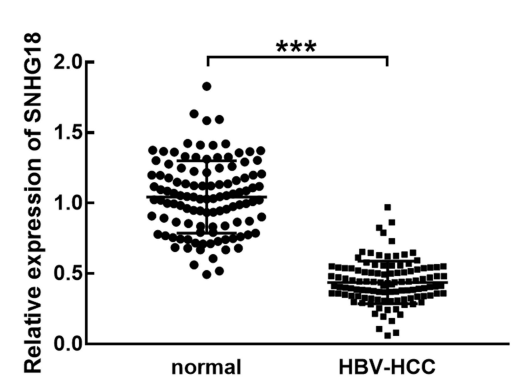

B

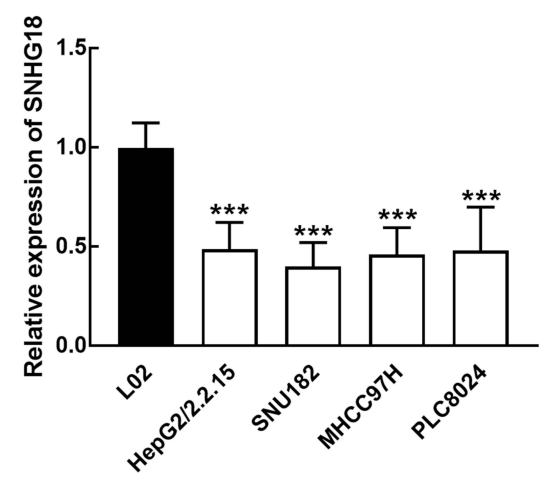

C

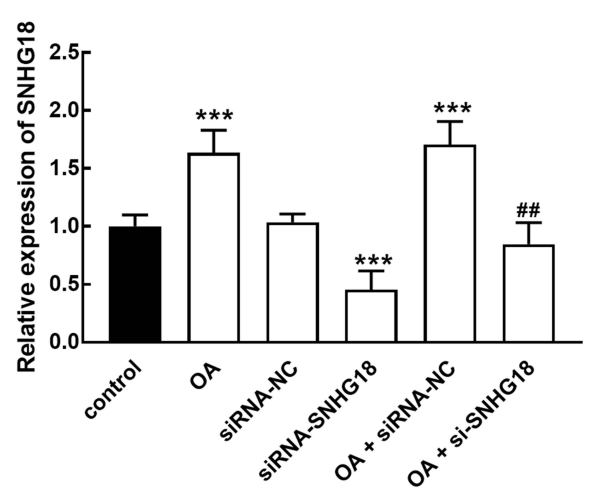

Figure 2 SNHGI 8 expression in HBV-associated HCC. (A and B) The significant downregulation of SNHGI8 was observed in HBV-associated HCC tissues (A) and cells (B). (C) OA dramatically enhanced the expression of SNHGI8 and reversed its knockdown induced by siRNA transfection. $* * * p<0.00 \mathrm{I}$ relatives to the normal tissues and cells. ${ }^{\#} p<0.01$ relative to the transfection of siRNA-SNHGI 8. 


\section{SNHGI8 Expression Evaluation}

The expression of SNHG18 was estimated by quantitative PCR with the help of Bio-Rad CFX96 (Bio-Rad Lab, USA) using SYBR Green. Total RNA was extracted and reversed to cDNA with Superscript II (Thermo Scientific, USA). The SNHG18 expression level was calculated by the $2^{-\Delta \Delta C T}$ method with GAPDH as the internal reference.

\section{HBV-Associated HCC Tissues}

One hundred and eleven pairs of tumor and matched normal tissues were obtained from HBV-infected HCC patients. All patients were diagnosed and received surgical treatment at The First Affiliated Hospital of Jiamusi University from 20132015. The included patients had never received any antitumor therapy before the enrollment. The collected tissues were stored in liquid nitrogen and frozen at $-80^{\circ} \mathrm{C}$ until further analyses. Informed consent was obtained from all individual participants included in the study and all patients were followed up for 5 years to obtain their survival status. This study was performed according to the principles of the Declaration of Helsinki and approved by the Ethics Committee of The First Affiliated Hospital of Jiamusi University.

Table I Association Between IncRNA SNHGI8 and Clinicopathological Features of HBV-Associated HCC Patients

\begin{tabular}{|c|c|c|c|c|}
\hline & \multirow[t]{2}{*}{ Cases $(n=I I I)$} & \multicolumn{2}{|c|}{ Expression of IncRNA SNHG I 8} & \multirow[t]{2}{*}{$P$ value } \\
\hline & & High $(n=53)$ & Low $(n=58)$ & \\
\hline Age (years) & & & & 0.644 \\
\hline$\leq 55$ & 54 & 27 & 27 & \\
\hline$>55$ & 57 & 26 & 31 & \\
\hline Gender & & & & 0.347 \\
\hline Male & 74 & 33 & 41 & \\
\hline Female & 37 & 20 & 17 & \\
\hline $\operatorname{AFP}(\mathrm{ng} / \mathrm{mL})$ & & & & $0.012^{*}$ \\
\hline$\leq 20$ & 53 & 27 & 26 & \\
\hline$>20$ & 58 & 26 & 32 & \\
\hline Tumor size $(\mathrm{cm})$ & & & & 0.155 \\
\hline$\leq 5$ & 55 & 30 & 25 & \\
\hline$>5$ & 56 & 23 & 33 & \\
\hline TNM stage & & & & $0.027^{*}$ \\
\hline I-II & 79 & 43 & 36 & \\
\hline III & 32 & 10 & 22 & \\
\hline Differentiation & & & & 0.182 \\
\hline Well + moderate & 77 & 40 & 37 & \\
\hline Poor & 34 & 13 & 21 & \\
\hline Liver cirrhosis & & & & 0.252 \\
\hline Negative & 30 & 17 & 13 & \\
\hline Positive & 81 & 36 & 45 & \\
\hline
\end{tabular}

Notes: $* P<0.05$ indicates the significant association.

Abbreviation: AFP, $\alpha$-fetoprotein. 


\section{Statistical Analysis}

All experiments were conducted in triplicate independent repeats. Obtained data were presented as mean value \pm SD. and analyzed by GraphPad Primer 7.0 (GraphPad, USA). The differences were evaluated with one-way ANOVA followed by Turkey's post-hoc test. Kaplan-Meier and multivariate Cox regression analysis were used to conduct the prognostic evaluation. $p<0.05$ indicated a statistically significant difference.

\section{Results}

\section{OA Inhibited Cellular Processes of HBV-HCC}

In the typical HBV-associated HCC cells, the incubation with OA significantly suppressed HepG2/2.2.15 and MHCC97H cell proliferation $(p<0.05, p<0.01$, Figure 1A). Meanwhile, the significant inhibitory effect of OA was also exerted on HepG2/2.2.15 and MHCC97H cell migration (Figure 1B) and invasion (Figure $1 \mathrm{C}, p<0.05, p<0.01, p<0.001$ ), and the inhibitory effect was enhanced with the increasing concentration of OA.

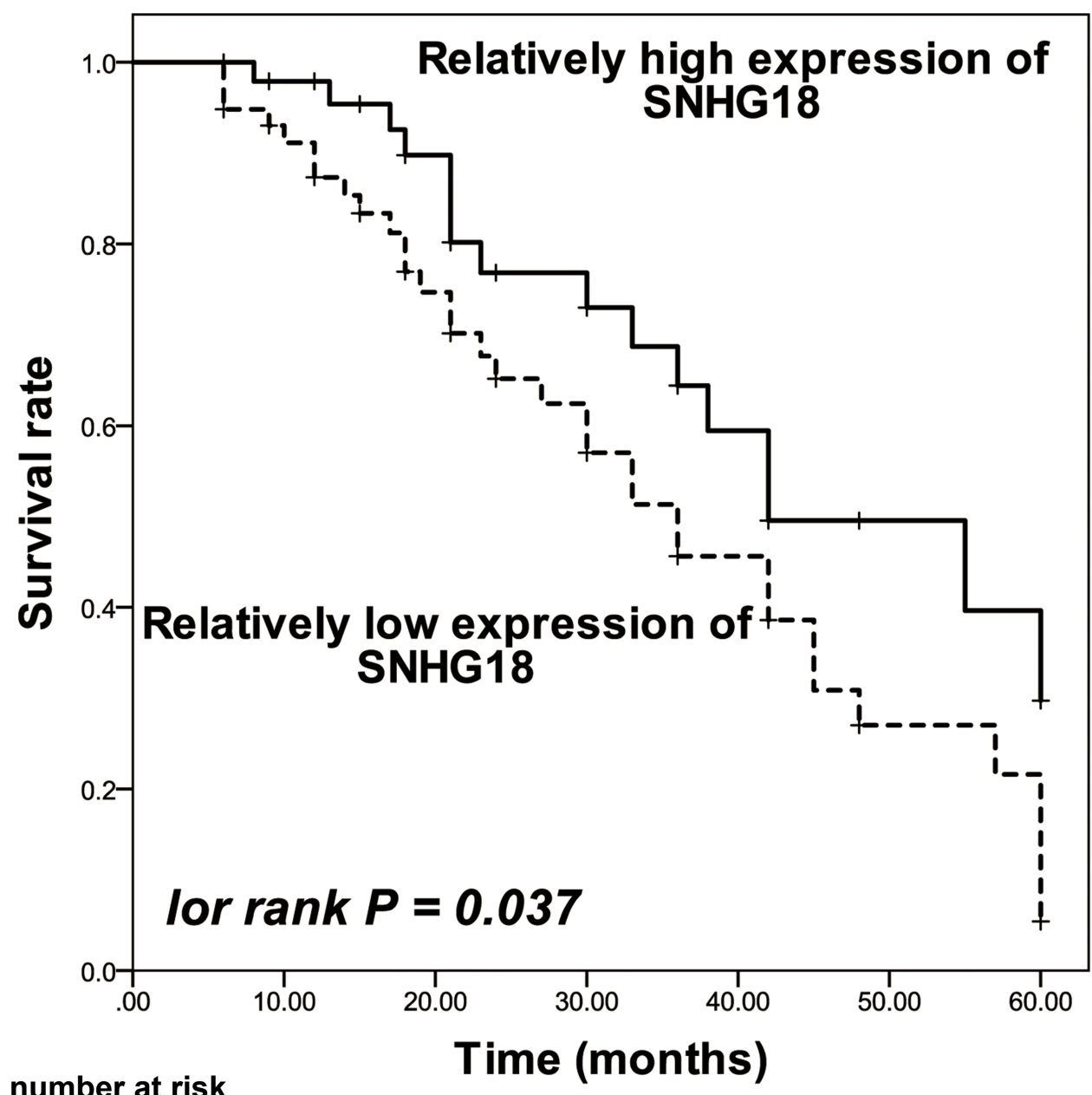

\section{high SNHG18}

$\begin{array}{llllllllll}48 & 43 & 39 & 34 & 28 & 24 & 20 & 13 & 9 & 5\end{array}$

low SNHG18

$\begin{array}{llllllllll}53 & 49 & 44 & 39 & 33 & 28 & 24 & 20 & 14 & 8\end{array}$

Figure 3 Kaplan-Meier curve of enrolled patients based on the average expression of SNHGI8 in collected tumor tissues. Patients with a relatively low expression of SNHGI 8 showed a poorer survival rate in comparison with patients with relatively high SNHGI 8 expression. $\log$ rank $P=0.037$. 
Table 2 Prognostic Value of Clinicopathological Features Evaluated by Multiple Cox Regression Analysis

\begin{tabular}{|l|l|l|l|}
\hline & HR Value & $95 \% \mathbf{C l}$ & P value \\
\hline LncRNA SNHGI8 & 2.701 & $1.306-5.585$ & $0.007^{* * *}$ \\
\hline Age & 1.259 & $0.705-2.248$ & 0.437 \\
\hline Gender & 1.426 & $0.760-2.675$ & 0.269 \\
\hline AFP & 1.662 & $0.854-3.236$ & 0.135 \\
\hline Tumor size & 1.726 & $0.904-3.294$ & 0.098 \\
\hline TNM stage & 2.123 & $1.148-3.926$ & $0.016 *$ \\
\hline Differentiation & 1.633 & $0.820-3.251$ & 0.163 \\
\hline Liver cirrhosis & 1.767 & $0.881-3.547$ & 0.109 \\
\hline
\end{tabular}

Notes: $* P<0.05 * * * P<0.001$ indicates the significantly prognostic value.

Abbreviation: AFP, $\alpha$-fetoprotein.

\section{OA Elevated the Expression of SNHGI8 in HBV-HCC}

In collected HBV- HCC tissues, the level of SNHG18 was significantly lower than that in normal tissues $(p<0.001$, Figure 2A). Consistently, the downregulation of SNHG18 was also found in HBV-associated HCC cells relative to normal L02 cells $(p<0.001$, Figure 2B). In HepG2/2.2.15 cell, the transfection of siRNA-SNHG18 exerted an inhibition on SNHG18 expression, while the treatment with $30 \mu \mathrm{g} / \mathrm{mL}$ OA dramatically enhanced SNHG18 expression compared with untreated cells, which was reversed by the transfection of si-SNHG18 $(p<0.01, p<0.001$, Figure 2C).

\section{SNHGI 8 Serves as a Biomarker of HBV-HCC}

The enrolled patients were grouped according to the average expression of SNHG18 in tumor tissues. The high SNHG18 group included 33 males and 20 females and the low SNHG18 group included 58 patients (41 males and 17 females). Among major clinicopathological characteristics of patients, the AFP levels $(p=0.012)$ and the TNM stage $(p=0.027)$ of patients were found to be closely associated with the expression of SNHG18 (Table 1).

Furthermore, the overall survival of patients with relatively low SNHG18 level was much poorer than patients with relatively high SNHG18 level (log rank $p=0.037$, Figure 3). SNHG18 was alsoan independent prognostic biomarker of HBV-associated HCC with a HR value of $2.701(95 \% \mathrm{CI}=1.306-5.585, p=0.007)$ as well as TNM with the HR value of $2.123(95 \% \mathrm{CI}=1.148-3.926, p=0.016$, Table 2$)$.

\section{SNHG 8 Mediates the Antitumor Effect of OA in HCC}

In HBV- HCC cells, the knockdown of SNHG18 showed a significant promoted effect on critical processes, including cell proliferation (Figure 4A), migration (Figure 4B), and invasion (Figure 4C) compared with untransfected cells $(p<$ $0.05, p<0.01)$. Meanwhile, the downregulation of SNHG18 was found to attenuate the antitumor effect of OA on HepG2/2.2.15 and MHCC97H cells $(p<0.01$, Figure 4A-C).

\section{Discussion}

The antitumor effect of $\mathrm{OA}$ has been widely reported in colorectal cancer, non-small cell lung cancer, and breast cancer. ${ }^{10,15,16}$ In HCC, the inhibitory effect of OA on tumor progression has also been uncovered, ${ }^{7,8}$ but the molecular mechanism has not been explored yet. The suppressed effect of OA on tumor progression of HBV-associated HCC was observed, which is consistent with former studies. ${ }^{7,8}$ Meanwhile, the inhibitory effect of OA was found to perform in a concentration-dependent manner, which was enhanced with the increasing concentration. 


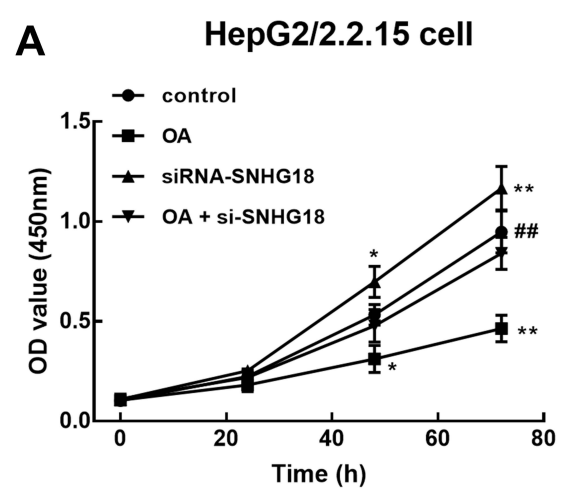

B

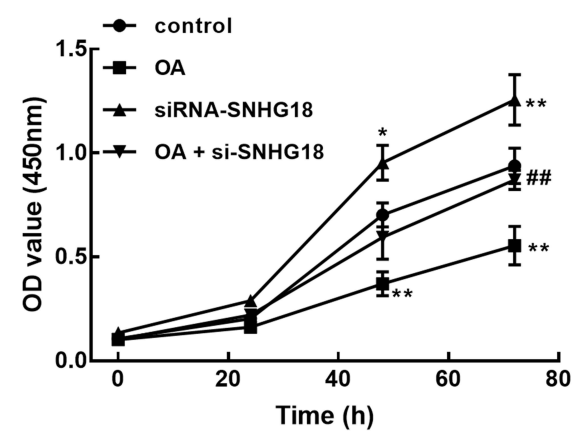

HepG2/2.2.15 cell

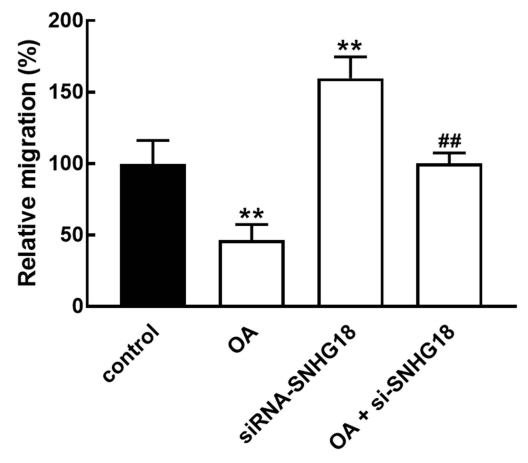

MHCC97H cell

C

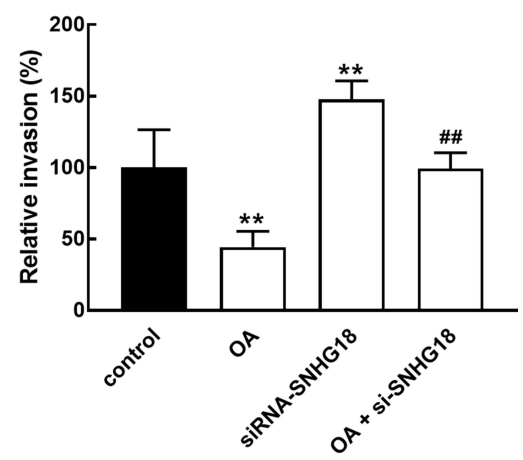

MHCC97H cell
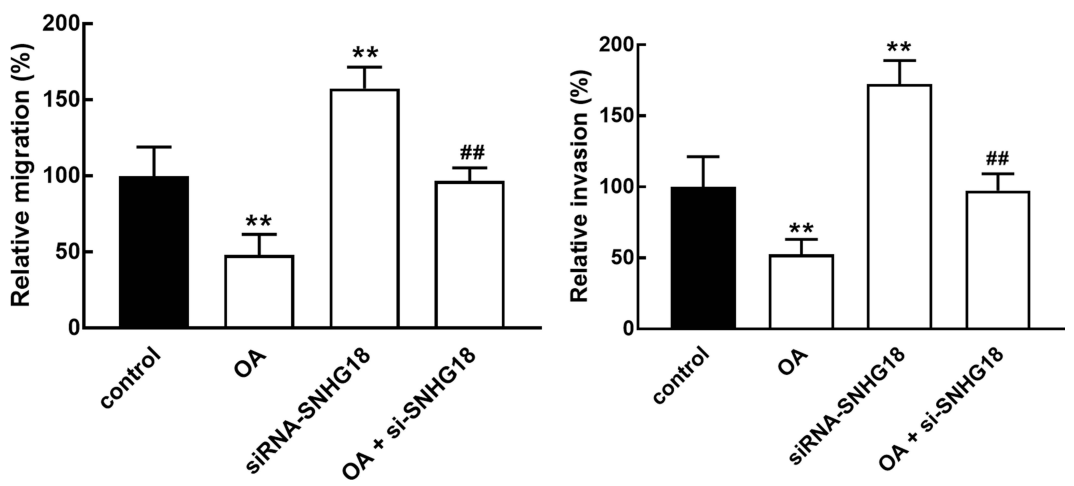

Figure 4 SNHGI8 mediated the antitumor effect of OA in HBV-associated HCC. SNHGI8 acted as a tumor suppressor of HBV-associated HCC, of which the downregulation exerted significantly promoted effect on cell proliferation (A), migration (B), and invasion (C). While the knockdown of SNHGI 8 was able to alleviate the inhibition of $\mathrm{HBV}$-associated $\mathrm{HCC}$ by $\mathrm{OA}$. ${ }^{*} p<0.05$, ${ }^{* *} p<0.0$ I relative to the control group. ${ }^{\#} p<0.0 \mathrm{I}$ relative to the treatment of $\mathrm{OA}(30 \mu \mathrm{g} / \mathrm{mL})$.

Previously, OA was suggested to enhance miR-421 in pancreatic cancer, which was considered as the molecular mechanism underlying the antitumor effect of OA. ${ }^{17}$ The JNK/mTOR signaling pathway was illustrated to participate in the protective effect of $\mathrm{OA}$ on autophagy in lung cancer, breast cancer, osteosarcoma, pancreatic cancer, and prostate cancer cells. ${ }^{18}$ Here, OA was revealed to regulate the expression of SNHG18 in HBV-associated HCC cells, which was hypothesized to be the potential mechanism of the inhibition of HCC development. Due to the high replication rates and viral mutations, HBV infection has been considered as a serious health problem and the postoperative survival rate of HBV-associate HCC patients is pessimistic. ${ }^{19,20}$ Improving the monitoring of the development of HBV-associated HCC remains a challenge in its clinical therapy. Recently, a number of biomarkers have been leaked out in tumor research. For example, lncRNA PCNAP1 promoted HBV replication via interacting with HBV ccDNA and therefore drive tumor progression and hepatocarcinogenesis. ${ }^{21}$ The involvement of SNHG18 was disclosed in glioma, due to the fact that SNHG18 could enhance the radioresistance of glioma cells and promote cell migration and invasion. ${ }^{14,22}$ Similarly, SNHG18 was not only associated with the severity and progression of osteosarcoma, but it was also able to predict the poor prognosis of patients. ${ }^{13}$ Herein, the significant downregulation of SNHG18 was observed in HBV-associated HCC, which is consistent with the previous assessment of SNHG18 expression in HCC. ${ }^{12}$ The reduced expression of SNHG18 showed a close relationship with the high AFP level and advanced TNM stage of patients, which are an indicator of the malignant progression of HCC, indicating the potential involvement of HBV-associated HCC of SNHG18. Additionally, SNHG18 was also found to be significantly related to the overall survival and was appraised as an independent prognostic indicator of HBV-associated HCC together with the TNM stage. 
Moreover, SNHG18 was also observed to exert inhibitory effects on major cellular processes of HBV-associated HCC, including cell growth, metastasis, and invasion, suggesting its tumor suppressor role. OA elevated the expression level of SNHG18, while SNHG18 could also alleviate the inhibition of HBV-associated HCC development by OA. Therefore, the antitumor effect of OA in HBV-associated HCC was speculated to be a result of the enhancement of SNHG18, which suppressed tumor progression.

However, the relatively small sample size of this study compared with previous data from the public databases might limit the results. The insignificant association between SNHG18 and some patients' critical clinicopathological features, such as tumor size, differentiation, and liver cirrhosis. ${ }^{23-29}$ These characteristics of patients are also crucial for the disease development and the clinical outcome of patients, the unobvious significance of these features might result from the limited sample size. On the other hand, the lack of in vivo validation is also a prospect of this study in further investigations.

\section{Conclusion}

Taken together, SNHG18 participated in the development of HBV-associated HCC and predicted the prognosis of patients. OA showed a dramatic antitumor effect on the cellular processes of HBV-associated HCC and increased the expression of SNHG18. SNHG18 was demonstrated as a tumor suppressor mediating the antitumor effect of OA in HBVassociated HCC.

\section{Funding}

The present study was supported by The Team of Jiamusi University Biology (Grant No.jdxktd2019003), The screening of new anti-tumor targets and drug designing (Grant No.jcyxhxkt2021-01), Horizontal subject of Jiangsu Vocational College of Medicine, Key Laboratory of Microecology-immune Regulatory Network and Related Diseases Open Project (Grant No.2021-SZD-JC-001).

\section{Disclosure}

The authors report no conflicts of interest in this work.

\section{References}

1. Chen Y, Tian Z. HBV-induced immune imbalance in the development of HCC. Front Immunol. 2019;10:2048. doi:10.3389/fimmu.2019.02048

2. Xie Y. Hepatitis B virus-associated hepatocellular carcinoma. Adv Exp Med Biol. 2017;1018:11-21.

3. Luo P, Wu S, Yu Y, et al. Current status and perspective biomarkers in AFP negative HCC: towards screening for and diagnosing hepatocellular carcinoma at an earlier stage. Pathol Oncol Res. 2020;26(2):599-603. doi:10.1007/s12253-019-00585-5

4. Wang W, Wei C. Advances in the early diagnosis of hepatocellular carcinoma. Genes Dis. 2020;7(3):308-319. doi:10.1016/j.gendis.2020.01.014

5. Pollier J, Goossens A. Oleanolic acid. Phytochemistry. 2012;77:10-15. doi:10.1016/j.phytochem.2011.12.022

6. Zhou R, Zhang Z, Zhao L, et al. Inhibition of mTOR signaling by oleanolic acid contributes to its anti-tumor activity in osteosarcoma cells. J Orthop Res. 2011;29(6):846-852. doi:10.1002/jor.21311

7. Wang $\mathrm{H}$, Zhong $\mathrm{W}$, Zhao J, et al. Oleanolic acid inhibits epithelial-mesenchymal transition of hepatocellular carcinoma by promoting iNOS dimerization. Mol Cancer Ther. 2019;18(1):62-74. doi:10.1158/1535-7163.MCT-18-0448

8. Zhou W, Zeng X, Wu X. Effect of oleanolic acid on apoptosis and autophagy of SMMC-7721 hepatoma cells. Med Sci Monit. $2020 ; 26:$ e921606. doi:10.12659/MSM.921606

9. Chaturvedi VK, Singh A, Dubey SK, Hetta HF, John J, Singh MP. Molecular mechanistic insight of hepatitis B virus mediated hepatocellular carcinoma. Microb Pathog. 2019;128:184-194. doi:10.1016/j.micpath.2019.01.004

10. Zhao X, Liu M, Li D. Oleanolic acid suppresses the proliferation of lung carcinoma cells by miR-122/Cyclin G1/MEF2D axis. Mol Cell Biochem. 2015;400(1-2):1-7. doi:10.1007/s11010-014-2228-7

11. Han D, Zhang X, Zhang J, et al. Oleanolic acid suppresses vascular smooth muscle cell proliferation by increasing lincRNA-p21 expression. Oncol Lett. 2016;12(5):3519-3522. doi:10.3892/ol.2016.5096

12. Liu XF, Thin KZ, Ming XL, et al. Small nucleolar RNA host gene 18 acts as a tumor suppressor and a diagnostic indicator in hepatocellular carcinoma. Technol Cancer Res Treat. 2018;17:1533033818794494. doi:10.1177/1533033818794494

13. Huang LJ, Shen Y, Bai J, et al. High expression levels of long noncoding RNA small nucleolar RNA host gene 18 and semaphorin 5A indicate poor prognosis in multiple myeloma. Acta Haematol. 2020;143(3):279-288. doi:10.1159/000502404

14. Zheng R, Yao Q, Ren C, et al. Upregulation of long noncoding RNA small nucleolar RNA host gene 18 promotes radioresistance of glioma by repressing semaphorin 5A. Int J Radiat Oncol Biol Phys. 2016;96(4):877-887. doi:10.1016/j.ijrobp.2016.07.036

15. Bao Y, Zhang S, Chen Z, et al. Synergistic chemotherapy for breast cancer and breast cancer brain metastases via paclitaxel-loaded oleanolic acid nanoparticles. Mol Pharm. 2020;17(4):1343-1351. doi:10.1021/acs.molpharmaceut.0c00044 
16. Niu G, Sun L, Pei Y, Wang D. Oleanolic acid inhibits colorectal cancer angiogenesis by blocking the VEGFR2 signaling pathway. Anticancer Agents Med Chem. 2018;18(4):583-590. doi:10.2174/1871520617666171020124916

17. Shopit A, Li X, Tang Z, et al. miR-421 up-regulation by the oleanolic acid derivative K73-03 regulates epigenetically SPINK1 transcription in pancreatic cancer cells leading to metabolic changes and enhanced apoptosis. Pharmacol Res. 2020;161:105130. doi:10.1016/j.phrs.2020.105130

18. Liu J, Zheng L, Zhong J, Wu N, Liu G, Lin X. Oleanolic acid induces protective autophagy in cancer cells through the JNK and mTOR pathways. Oncol Rep. 2014;32(2):567-572. doi:10.3892/or.2014.3239

19. Li YW, Yang FC, Lu HQ, Zhang JS. Hepatocellular carcinoma and hepatitis B surface protein. World J Gastroenterol. 2016;22(6):1943-1952. doi:10.3748/wjg.v22.i6.1943

20. Tong S, Revill P. Overview of hepatitis B viral replication and genetic variability. J Hepatol. 2016;64(1 Suppl):S4-S16. doi:10.1016/j. jhep.2016.01.027

21. Feng J, Yang G, Liu Y, et al. LncRNA PCNAP1 modulates hepatitis B virus replication and enhances tumor growth of liver cancer. Theranostics. 2019;9(18):5227-5245. doi:10.7150/thno.34273

22. Zheng R, Yao Q, Li X, Xu B. Long noncoding ribonucleic acid SNHG18 promotes glioma cell motility via disruption of alpha-enolase nucleocytoplasmic transport. Front Genet. 2019;10:1140. doi:10.3389/fgene.2019.01140

23. Fattovich G, Stroffolini T, Zagni I, Donato F. Hepatocellular carcinoma in cirrhosis: incidence and risk factors. Gastroenterology. 2004;127(5 Suppl 1):S35-S50. doi:10.1053/j.gastro.2004.09.014

24. Kanda T, Goto T, Hirotsu Y, Moriyama M, Omata M. Molecular mechanisms driving progression of liver cirrhosis towards hepatocellular carcinoma in chronic hepatitis B and C infections: a review. Int J Mol Sci. 2019;20(6):1358. doi:10.3390/ijms20061358

25. Daoud A, Teeter L, Ghobrial RM, et al. Transplantation for hepatocellular carcinoma: is there a tumor size limit? Transplant Proc. 2018;50 (10):3577-3581. doi:10.1016/j.transproceed.2018.04.038

26. Huang WJ, Jeng YM, Lai HS, Sheu FY, Lai PL, Yuan RH. Tumor size is a major determinant of prognosis of resected stage I hepatocellular carcinoma. Langenbecks Arch Surg. 2015;400(6):725-734. doi:10.1007/s00423-015-1329-4

27. Kim SJ, Lee KK, Kim DG. Tumor size predicts the biological behavior and influence of operative modalities in hepatocellular carcinoma. Hepatogastroenterology. 2010;57(97):121-126.

28. Liu M, Yan Q, Sun Y, et al. A hepatocyte differentiation model reveals two subtypes of liver cancer with different oncofetal properties and therapeutic targets. Proc Natl Acad Sci U S A. 2020;117(11):6103-6113. doi:10.1073/pnas.1912146117

29. Zhang W, Mu D, Feng K. Hierarchical potential differentiation of liver cancer stem cells. Adv Clin Exp Med. 2017;26(7):1137-1141. doi:10.17219/ acem $/ 66343$

Cancer Management and Research is an international, peer-reviewed open access journal focusing on cancer research and the optimal use of preventative and integrated treatment interventions to achieve improved outcomes, enhanced survival and quality of life for the cancer patient. The manuscript management system is completely online and includes a very quick and fair peer-review system, which is all easy to use. Visit http://www.dovepress.com/testimonials.php to read real quotes from published authors. 